\title{
Constraints on coloured scalars from global fits
}

\author{
Otto Eberhardt, Víctor Miralles and Antonio Pich \\ Instituto de Física Corpuscular, Parque Científico, \\ C/Catedrático José Beltrán, 2, E-46980 Paterna, Spain \\ E-mail: otto.eberhardt.physics@gmail.com, victor.miralles@ific.uv.es, \\ antonio.pich@ific.uv.es
}

ABSTRACT: We consider a simple extension of the electroweak theory, incorporating one $\mathrm{SU}(2)_{L}$ doublet of colour-octet scalars with Yukawa couplings satisfying the principle of minimal flavour violation. Using the HEPfit package, we perform a global fit to the available data, including all relevant theoretical constraints, and extract the current bounds on the model parameters. Coloured scalars with masses below $1.05 \mathrm{TeV}$ are already excluded, provided they are not fermiophobic. The mass splittings among the different (charged and CP-even and CP-odd neutral) scalars are restricted to be smaller than $20 \mathrm{GeV}$. Moreover, for scalar masses smaller than $1.5 \mathrm{TeV}$, the Yukawa coupling of the coloured scalar multiplet to the top quark cannot exceed the one of the SM Higgs doublet by more than $80 \%$. These conclusions are quite generic and apply in more general frameworks (without fine tunings). The theoretical requirements of perturbative unitarity and vacuum stability enforce relevant constraints on the quartic scalar potential parameters that are not yet experimentally tested.

KeYwords: Beyond Standard Model, Higgs Physics

ArXiv EPrint: 2106.12235 


\section{Contents}

1 Introduction 1

2 The scalar colour-octet model 2

3 Fit constraints 3

3.1 Theoretical constraints 4

3.2 Higgs constraints 5

3.3 Constraints from direct searches 6

3.4 Flavour constraints

$\begin{array}{lll}3.5 & \text { Electroweak precision data } & 7\end{array}$

4 Results $\quad 8$

4.1 Theoretical constraints 8

$\begin{array}{lll}4.2 & \text { Experimental constraints } & 10\end{array}$

$\begin{array}{lll}4.3 & \text { All constraints } & 12\end{array}$

$\begin{array}{lll}5 & \text { Summary } & 15\end{array}$

\section{Introduction}

The main focus of the first LHC run was on the search for the Standard Model (SM) Higgs boson and culminated in its spectacular discovery in 2012 [1,2]. After the conclusion of the run 2, the properties of this new scalar are much better known, and they seem to agree very well with the SM predictions. In combination with direct LHC searches for additional spin-0 particles, they put strong bounds on extensions of the scalar sector of the SM, as for example the Two-Higgs-Doublet model [3]. On the other hand, new physics beyond the Standard Model (BSM) is needed to fix the shortcomings of the current theoretical framework, and many BSM scenarios could manifest themselves through the appearance of additional scalars at the LHC. Most of these SM extensions contain scalar fields which transform as singlets under the $\mathrm{SU}(3)_{C}$ group of $\mathrm{QCD}$ and, therefore, do not carry colour charge. But coloured scalars are less constrained than one might naively think: they do not mix with the SM Higgs and contribute to its signal strengths only at the loop level. However, recent LHC searches for scalar resonances are expected to yield strong constraints on their properties.

Here we want to study a simple extension of the SM scalar sector with one $\mathrm{SU}(2)_{L}$ doublet of scalar fields that transforms as an octet under the colour $\mathrm{SU}(3)_{C}$ group. In some sense, one can think of it as a Two-Higgs-Doublet model in which one of the doublets is coloured. Scalars of this type with masses around the electroweak scale could emerge naturally from $\mathrm{SU}(5)$ or $\mathrm{SO}(10)$ unification theories at high energy scales [4-7]. As this 
model was first proposed by Manohar and Wise [8], we will refer to it as the ManoharWise (MW) model in the following. Several phenomenological aspects of this dynamical scenario have been studied in the literature [9-29], yet a comprehensive analysis including all relevant up-to-date constraints has not been performed.

In this article we will present such an analysis. After defining the MW model in section 2, we briefly describe in section 3 the HEPfit package [30] that we employ to perform the global fits. This section explains in detail the various constraints on the parameter space of the model, which come from theoretical considerations (section 3.1), Higgs measurements (section 3.2), direct LHC searches (section 3.3) and flavour observables (section 3.4). Putting all pieces together, we show the results of our fits in section 4. Finally, section 5 contains a summary of our findings and a brief outlook on future prospects.

\section{The scalar colour-octet model}

The MW model extends the SM with one electroweak doublet of colour-octet scalar fields with hypercharge $Y=\frac{1}{2}$. Since it has colour charge, the new scalar multiplet does not mix with the SM Higgs doublet. Furthermore, the coloured particles cannot acquire a vacuum expectation value (vev) because colour must be conserved. Therefore, only the SM Higgs boson will acquire a vev which will minimise the most general potential that can be built with this scalar sector:

$$
\begin{aligned}
V_{\mathrm{MW}}= & m_{\Phi}^{2} \Phi^{\dagger} \Phi+\frac{1}{2} \lambda\left(\Phi^{\dagger} \Phi\right)^{2}+2 m_{S}^{2} \operatorname{Tr}\left(S^{\dagger i} S_{i}\right)+\mu_{1} \operatorname{Tr}\left(S^{\dagger i} S_{i} S^{\dagger j} S_{j}\right)+\mu_{2} \operatorname{Tr}\left(S^{\dagger i} S_{j} S^{\dagger j} S_{i}\right) \\
& +\mu_{3} \operatorname{Tr}\left(S^{\dagger i} S_{i}\right) \operatorname{Tr}\left(S^{\dagger j} S_{j}\right)+\mu_{4} \operatorname{Tr}\left(S^{\dagger i} S_{j}\right) \operatorname{Tr}\left(S^{\dagger j} S_{i}\right)+\mu_{5} \operatorname{Tr}\left(S_{i} S_{j}\right) \operatorname{Tr}\left(S^{\dagger i} S^{\dagger j}\right) \\
& +\mu_{6} \operatorname{Tr}\left(S_{i} S_{j} S^{\dagger j} S^{\dagger i}\right)+\nu_{1} \Phi^{\dagger i} \Phi_{i} \operatorname{Tr}\left(S^{\dagger j} S_{j}\right)+\nu_{2} \Phi^{\dagger i} \Phi_{j} \operatorname{Tr}\left(S^{\dagger j} S_{i}\right) \\
& +\left[\nu_{3} \Phi^{\dagger i} \Phi^{\dagger j} \operatorname{Tr}\left(S_{i} S_{j}\right)+\nu_{4} \Phi^{\dagger i} \operatorname{Tr}\left(S^{\dagger j} S_{j} S_{i}\right)+\nu_{5} \Phi^{\dagger i} \operatorname{Tr}\left(S^{\dagger j} S_{i} S_{j}\right)+\text { h.c. }\right]
\end{aligned}
$$

where $\Phi=\left(\phi^{+}, \phi^{0}\right)^{T}$ is the usual SM doublet, the traces are taken in colour space, and $i$ and $j$ denote $\mathrm{SU}(2)_{L}$ indices. The additional $(8,2)_{1 / 2}$ scalar fields $S^{A}=\left(S^{A,+}, S^{A, 0}\right)^{T}$ are contained in the multiplet $S=S^{A} T^{A}$ with $T^{A}$ the generators of the $\mathrm{SU}(3)_{C}$ group. All potential parameters are real except $\nu_{3}, \nu_{4}$ and $\nu_{5}$, but performing a phase rotation we can always take $\nu_{3}$ to be also real.

From eq. (2.1) we can derive the masses of the physical neutral octet scalar $\left(m_{R}\right)$, the neutral octet pseudoscalar $\left(m_{I}\right)$ and the charged octet $\left(m_{S^{ \pm}}\right)$, which are split by the Higgs vev, $\left\langle\phi^{0}\right\rangle=v / \sqrt{2}$ :

$$
m_{S^{ \pm}}^{2}=m_{S}^{2}+\nu_{1} \frac{v^{2}}{4}, \quad m_{R, I}^{2}=m_{S}^{2}+\left(\nu_{1}+\nu_{2} \pm 2 \nu_{3}\right) \frac{v^{2}}{4} .
$$

The kinetic term (the factor two gives the correct canonical normalisation for the fields)

$$
\mathcal{L}_{K}=2 \operatorname{Tr}\left[\left(D_{\mu} S\right)^{\dagger} D^{\mu} S\right]
$$

generates the interaction of the octet scalars with the gauge bosons through the covariant derivative

$$
D_{\mu} S=\partial_{\mu} S+i g_{s}\left[G_{\mu}, S\right]+i g \frac{\sigma^{i}}{2} W_{\mu}^{i} S+\frac{i}{2} g^{\prime} B_{\mu} S,
$$


with $G_{\mu}=G_{\mu}^{A} T^{A}$ the octet gluon field. Thus, these interactions are determined by the gauge symmetry and do not introduce additional free parameters.

The coloured scalars can also couple to the quarks through the Yukawa interaction. In order to guarantee the suppression of unwanted flavour-changing neutral currents, which are extremely suppressed experimentally, we will assume the principle of Minimal Flavour Violation (MFV) [31, 32], which is based on the hypothesis that all Yukawa matrices are proportional to the same flavour structures that break the $\mathrm{SU}(3)_{Q_{L}} \otimes \mathrm{SU}(3)_{u_{R}} \otimes \mathrm{SU}(3)_{d_{R}}$ symmetry in the SM. This is in fact one of the main motivations of the MW model because the only scalar representations that can couple to quarks and be compatible with this principle are the colour octet or singlet electroweak doublets [8]. With this assumption, the Yukawa couplings of the coloured scalars take the form

$$
\mathcal{L}_{Y} \supset-\sum_{i, j=1}^{3}\left[\eta_{D} Y_{i j}^{d} \bar{Q}_{L_{i}} S d_{R_{j}}+\eta_{U} Y_{i j}^{u} \bar{Q}_{L_{i}} \tilde{S} u_{R_{j}}+\text { h.c. }\right] .
$$

Here, $i$ and $j$ are family indices, $Y^{q}=\sqrt{2} M_{q} / v(q=u, d)$ denote the up and down SM Yukawa matrices and the tilde in the $S$ field indicates charge conjugation. The proportionality constants $\eta_{U}$ and $\eta_{D}$ are, in general, complex parameters.

Looking at eqs. (2.1) and (2.5), we observe that the MW model contains 18 more parameters than the SM, 14 of which are real while the other 4 are imaginary phases. In order to simplify the phenomenological analysis and to reduce the total number of free parameters we will only work in the CP-conserving limit. This assumption removes the imaginary parts of $\nu_{4}, \nu_{5}, \eta_{U}$ and $\eta_{D}$ and we end up with only 14 new free parameters.

\section{$3 \quad$ Fit constraints}

Our statistical data analysis will be based on a global Bayesian fit. We make use of the public HEPfit package [30], which is interfaced with the Bayesian Analysis Toolkit [33]. This code has been already applied to several BSM analyses, including the Two-HiggsDoublet model [3, 34-36] and the Georgi-Machacek model [37]. We have adapted the code, including the additional routines needed to study the MW model. These routines are also public and can be extended in future works to incorporate additional observables. In our fit we have only included observables that have been directly calculated with this model. Constraints obtained from existing bounds on higher-dimension operators ${ }^{1}$ are omitted, since we prefer to directly include in the fit the observables used to derive those constraints. Indeed, HEPfit has also been proven to be extremely reliable to constrain higher-dimension operators [39-41]. One of the key features of HEPfit is its independence of other codes at runtime, which provides a very fast framework for statistical analyses.

Since we will try to use in our fit all the available information, we did not have any previous constraints on the MW parameters. Therefore we decided to use a uniform distribution as a prior for the 14 free parameters of the MW model. The ranges adopted are

\footnotetext{
${ }^{1}$ See for instance ref. [38] (and references therein) where some particular colour-octet models are analysed. Owing to the MFV assumption which strongly suppresses the scalar couplings to light quarks, the $p p \rightarrow t \bar{t}$ analysis of ref. [38] is not relevant for the MW model.
} 


\begin{tabular}{|c|c|c|c|c|c|}
\hline Parameters & $m_{S}^{2}$ & $\nu_{n}$ & $\mu_{n}$ & $\eta_{U}$ & $\eta_{D}$ \\
\hline Priors & $\left(0.4^{2}, 1.5^{2}\right) \mathrm{TeV}^{2}$ & $(-10,10)$ & $(-10,10)$ & $(-5,5)$ & $(-20,20)$ \\
\hline
\end{tabular}

Table 1. Prior values of the fitted parameters.

shown in table 1. The range taken for $\eta_{U}$ follows from the assumption of a perturbative top Yukawa coupling, while larger values of $\eta_{D}$ are possible since $m_{b} \ll m_{t}$. The dependence of our results on the priors used turns out to be small, as long as these priors are reasonable. For instance, increasing the range of $m_{S}^{2}$ up to $2^{2} \mathrm{TeV}^{2}$ leads to the same limits, but we do not gain any further information since many of the direct-searches analyses included do not go beyond $1.5 \mathrm{TeV}$. For $\nu_{n}, \mu_{n}$ and $\eta_{U}$ the posterior probabilities are basically zero in the limits of the ranges considered and the same constraints are always found if this (reasonable) condition is satisfied. The only exception is $\eta_{D}$ which could not be constrained within the range considered. However, higher values of $\eta_{D}$ will always bring stronger limits on the other parameters. The parameter space is constrained by the theoretical and experimental requirements that we now discuss.

\subsection{Theoretical constraints}

Since we are interested in the LHC phenomenology, we will assume that the MW model is well defined up to the few $\mathrm{TeV}$ scale. As a consequence, the renormalisation group (RG) evolution should be stable between the electroweak scale and $5 \mathrm{TeV}$. By RG stability we mean the absence of Landau poles as well as the fact that the scalar potential $V_{\mathrm{Mw}}$ should be bounded from below at any of the considered scales. The RG equations (without Yukawa and gauge couplings) were taken from ref. [20] and a set of necessary positivity bounds was derived in ref. [25].

Another strong constraint on the quartic couplings of $V_{\mathrm{MW}}$ is the unitarity requirement that the two-to-two scattering processes of the scalar particles should not have a probability larger than 1. This condition is usually expressed in terms of the partial wave amplitudes $a_{j}$. Using their LO $\left(a_{j}^{(0)}\right)$ and NLO $\left(a_{j}^{(1)}\right)$ expressions [25], perturbative unitarity can be imposed at LO, NLO and at the so-called $\mathrm{NLO}+$ approximation [42] that includes the square of the NLO correction and therefore contains some, but not all, NNLO terms [25]:

$$
\left|a_{j}^{(0)}+\operatorname{Re}\left(a_{j}^{(1)}\right)\right|^{2} \leq \frac{1}{4} .
$$

Note also that the LO and NLO expressions for the partial wave amplitudes are only available in the large- $s$ approximation and should not be applied below a certain scale $\mu_{u}$, which we choose to be $\mu_{u}=1.5 \mathrm{TeV}$ as the current limits on the mass of the colour-octet scalars are around $1 \mathrm{TeV}[28]$.

On top of these unitarity bounds, we also impose perturbative behaviour of the quantum corrections and allow only for scenarios in which the NLO contributions to the partial wave amplitudes are smaller in magnitude than the LO term.

In the fits, we run up to 3 or $5 \mathrm{TeV}$ each parameter set, sampled at the electroweak scale, and control at each intermediate step if the parameters still comply with positivity 


\begin{tabular}{|c|c|c|c|c|c|c|c|}
\hline \multirow[t]{2}{*}{ Production } & \multirow[t]{2}{*}{ Decay } & Reference & $\begin{array}{c}L \\
{\left[\mathrm{fb}^{-1}\right]}\end{array}$ & $\begin{array}{c}\sqrt{s} \\
{[\mathrm{TeV}]}\end{array}$ & Reference & $\begin{array}{c}L \\
{\left[\mathbf{f b}^{-1}\right]}\end{array}$ & $\begin{array}{c}\sqrt{s} \\
{[\mathrm{TeV}]}\end{array}$ \\
\hline & & \multicolumn{3}{|c|}{ ATLAS } & \multicolumn{3}{|c|}{ CMS } \\
\hline \multirow{2}{*}{$\begin{array}{c}\mathrm{ggF} \\
\mathrm{VBF} \\
\mathrm{VH} \\
\mathrm{ttH}\end{array}$} & \multirow{2}{*}{$h \rightarrow \gamma \gamma$} & {$[43]$} & $4.5 / 20.3$ & $7 / 8$ & {$[44]$} & $5.1 / 19.7$ & $7 / 8$ \\
\hline & & {$[45]$} & 139 & 13 & {$[46]$} & 137 & 13 \\
\hline \multirow{10}{*}{$\operatorname{ggF}$} & \multirow{2}{*}{$\mu^{+} \mu^{-}$} & - & - & - & {$[47]$} & $5 / 20$ & $7 / 8$ \\
\hline & & [48] & 139 & 13 & {$[49]$} & 137 & 13 \\
\hline & \multirow{2}{*}{$\tau^{+} \tau^{-}$} & {$[50]$} & $4.5 / 20.3$ & $7 / 8$ & {$[51]$} & $4.9 / 19.7$ & $7 / 8$ \\
\hline & & {$[52]$} & 139 & 13 & [53] & 137 & 13 \\
\hline & \multirow{2}{*}{$W W$} & {$[54,55]$} & $25,4.5 / 20.3$ & $7 / 8$ & {$[56]$} & $4.9 / 19.4$ & $7 / 8$ \\
\hline & & {$[52]$} & 139 & 13 & [53] & 137 & 13 \\
\hline & \multirow{2}{*}{$Z_{\gamma}$} & {$[57]$} & $4.7 / 20.3$ & $7 / 8$ & {$[58]$} & $5 / 19.6$ & $7 / 8$ \\
\hline & & [59] & 139 & 13 & {$[60]$} & 35.9 & 13 \\
\hline & \multirow{2}{*}{$Z Z$} & [61] & $4.5 / 20.3$ & $7 / 8$ & [62] & $5.1 / 19.7$ & $7 / 8$ \\
\hline & & [63] & 139 & 13 & [53] & 137 & 13 \\
\hline
\end{tabular}

Table 2. Higgs signal strengths measured by ATLAS and CMS.

and (above $\mu_{u}$ ) perturbative unitarity. If this is not the case, the running is stopped and the corresponding cut-off scale is returned as an output.

\subsection{Higgs constraints}

In the MW model there are additional contributions to some of the production and decay channels of the SM Higgs boson, due to the presence of the colour-octet scalars. At the LHC, the SM Higgs boson is produced mainly through gluon fusion ( $\mathrm{ggF}$ ), vector boson fusion (VBF), associated production with a $t \bar{t}$ pair (tth) and associated production with vector bosons (Wh/Zh). The LO contribution to the first process is a one-loop amplitude already in the SM. As the coloured scalars couple to gluons and to the SM Higgs boson, they will contribute to the gluon-fusion production mode at the same order than the SM. Similarly, the decay of the SM Higgs boson to photons is also a one-loop process in the SM to which the new scalars contribute.

The LHC data for the Higgs physics are parametrized in terms of the Higgs signal strengths, which are defined as the measured cross section times branching ratio for a given production and decay Higgs channel, in units of the SM prediction. Table 2 compiles the experimental papers from which we have taken the values of the different Higgs signal strengths. Comparing the measured values of these observables with their theoretical predictions [22], we will try to constrain the parameters $\nu_{1}, \nu_{2}$ and $\nu_{3}$, which are directly related with the mass splittings of the new scalars in eq. (2.2). 


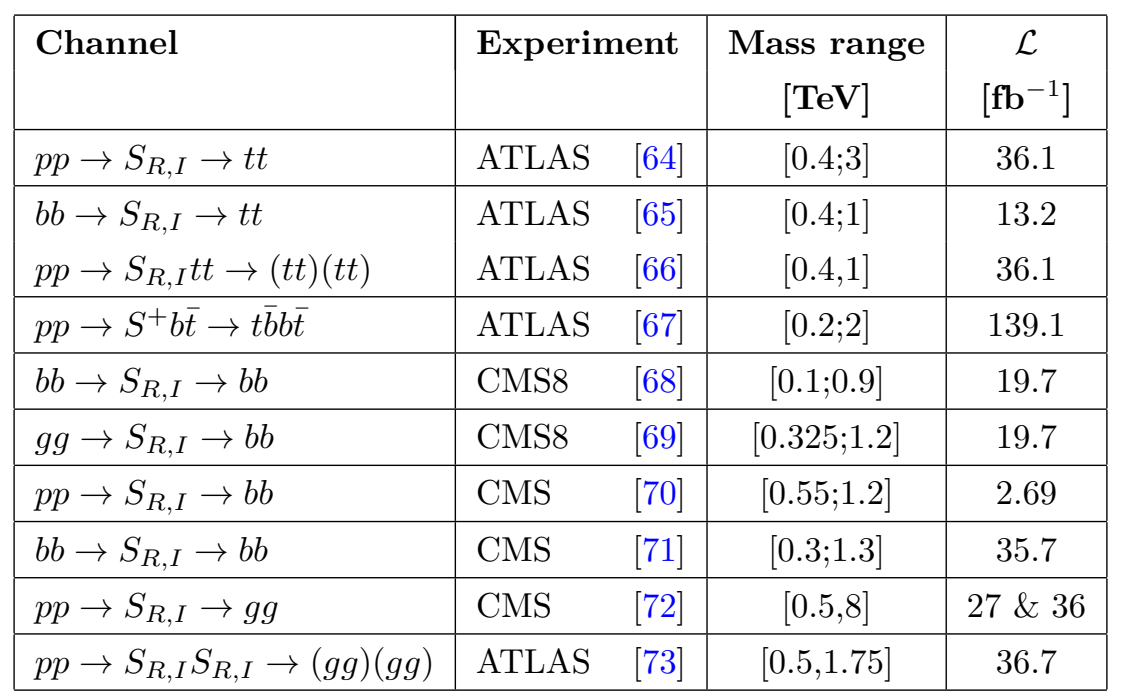

Table 3. Data from direct searches included in the fit. CMS8 means CMS at $8 \mathrm{TeV}$, the rest is at $13 \mathrm{TeV}$.

\subsection{Constraints from direct searches}

In order to find constraints from direct experimental searches of additional scalars, we will compare the theoretical prediction of the production cross-section times branching ratio of a given process, $\sigma \cdot \mathcal{B}$, with the experimental upper limits of the ATLAS and CMS collaborations. The channels that we have included in the fit are shown in table 3. Since decays of the colour-octet scalars into purely electroweak bosons are forbidden, we have only considered their decays into gluons and quarks. Furthermore, in order to be sure that the decay modes studied are indeed the dominant ones, we impose for each search that the mass difference between the coloured scalar we are analysing and the lightest member of the $S$ multiplet is smaller than the $W^{ \pm}$mass [28]. In this way, we forbid kinematically the decays into another coloured scalar and a gauge boson, which can become the dominant decay modes for some configurations of the parameter space. As we will show later, this assumption is well justified, given the constraints that perturbative unitarity enforce on the mass splittings of the new scalars.

Since HEPfit cannot generate events by itself, the theoretical predictions for $\sigma \cdot \mathcal{B}$ must be provided with other tools. We have used MadGraph [74] to create tables with the values of $\sigma \cdot \mathcal{B}$ for different input choices of the parameters on which these observables depend: $\eta_{U}, \eta_{D},\left(\nu_{4}+\nu_{5}\right)$ and $m_{S}$. These tables are read by HEPfit, which performs a linear interpolation to obtain the value of the observables at any point. The error of the linear interpolation is estimated to be $10 \%$ or less for $\log (\sigma \cdot \mathcal{B})$.

The experimental data are also provided in the form of tables, which compile the values of the $95 \%$ upper limits on $\sigma \cdot \mathcal{B}$, as a function of the resonance mass. In these tables, HEPfit also performs a linear interpolation if needed.

In order to compare the theoretical results with the experimental data, we define the ratio of the theoretical prediction over the experimental upper limit. To this ratio we will assign a Gaussian distribution (restricted to positive values) centered at 0 such that the value 1 is excluded with a $95 \%$ probability. 


\begin{tabular}{|c|c|c|}
\hline CKM Parameter & Input Value & Source \\
\hline$\left|V_{u d}\right|$ & $0.97370 \pm 0.00033$ & $\begin{array}{l}\text { Superallowed } 0^{+} \rightarrow 0^{+} \text {nuclear } \beta \\
\text { decays }\end{array}$ \\
\hline$\left|V_{u s}\right|$ & $0.2245 \pm 0.0008$ & $\begin{array}{l}\text { Kaon and pion semileptonic and leptonic } \\
\text { decays }\end{array}$ \\
\hline$\left|V_{c d}\right|$ & $0.221 \pm 0.004$ & \multirow{2}{*}{ Semileptonic and leptonic charm decays } \\
\hline$\left|V_{c s}\right|$ & $0.966 \pm 0.008$ & \\
\hline$\left|V_{c b}\right|$ & $(41.0 \pm 1.4) \times 10^{-3}$ & \multirow{2}{*}{$\begin{array}{l}\text { Exclusive and inclusive semileptonic } \\
\text { decays of } B \text { mesons }\end{array}$} \\
\hline$\left|V_{u b}\right|$ & $(3.82 \pm 0.24) \times 10^{-3}$ & \\
\hline$\left|V_{t d} / V_{t s}\right|$ & $0.205 \pm 0.006$ & $B^{0}-\bar{B}^{0}$ mixing \\
\hline
\end{tabular}

Table 4. Inputs $[75,76]$ used for the CKM fit and processes from which they are obtained. The error of $\left|V_{u d}\right|$ has been increased by a factor 2.4 .

\subsection{Flavour constraints}

Regarding flavour transitions, we have included in the analysis the $B_{s}^{0}-\bar{B}_{s}^{0}$ mixing and the decay $B_{s}^{0} \rightarrow \mu^{+} \mu^{-}$, which are the most constraining observables. The expressions for the relevant Wilson coefficients were taken from ref. [21], where a complete one-loop calculation was performed. Only the charged scalars contribute to these processes at the one-loop level, and the corresponding amplitudes involve the parameters $\eta_{U}$ and $\eta_{D}$. The leading $\eta_{U}$ contributions are proportional to the top-quark mass, while the $\eta_{D}$ terms are weighted by the bottom-quark mass. Therefore, the transition amplitudes are mainly governed by the parameter $\eta_{U}$.

These observables strongly depend on the numerical values of the relevant quark mixings. We cannot use the standard determinations of the CKM entries, since they assume the validity of the SM. In order to obtain non-contaminated values, we perform a specific CKM fit using HEPfit, which only includes observables that are not affected by the coloured scalars. The inputs of this CKM fit, shown in table 4, are taken from the PDG 2020 [75] except $\left|V_{c s}\right|$, for which we have adopted the more recent result of ref. [76]. The value of $\left|V_{u d}\right|$ has been considerably shifted with respect to the one quoted in the PDG 2018 [77]. Combined with the reduction of its uncertainty, this generates a violation of unitarity in the first row of the CKM matrix at the $3 \sigma$ level. Since there is no full consensus in the community, we have adopted a conservative attitude, increasing the error of $\left|V_{u d}\right|$ by a factor 2.4 so that CKM unitarity is recovered at $2 \sigma$, without modifying any central values. The results of this CKM fit, shown in table 5, are then used as inputs in our analysis of the MW parameter space.

\subsection{Electroweak precision data}

In this work, we are studying the most general CP-conserving MW model, so we have not imposed any additional assumptions such as Custodial Symmetry. Therefore, the coloured scalars generate non-vanishing contributions to the oblique parameters $[78,79]$ that can 


\begin{tabular}{|c|c|c|c|c|c|}
\hline & \multirow{2}{*}{ Value } & \multicolumn{4}{|c|}{ Correlation } \\
\cline { 3 - 6 } & & $\lambda$ & $A$ & $\bar{\rho}$ & $\bar{\eta}$ \\
\hline$\lambda$ & $0.22518 \pm 0.00069$ & 1 & -0.18 & 0.08 & -0.084 \\
$A$ & $0.808 \pm 0.028$ & -0.18 & 1 & -0.22 & -0.41 \\
$\bar{\rho}$ & $0.181 \pm 0.026$ & 0.08 & -0.22 & 1 & 0.039 \\
$\bar{\eta}$ & $0.360 \pm 0.029$ & -0.084 & -0.41 & 0.039 & 1 \\
\hline
\end{tabular}

Table 5. Wolfenstein parameters obtained from a fit to the CKM entries in table 4.

\begin{tabular}{|c|c|c|c|c|}
\hline \multirow{2}{*}{ Value } & \multicolumn{3}{|c|}{ Correlation } \\
\cline { 3 - 5 } & & $S$ & $T$ & $U$ \\
\hline$S$ & $0.093 \pm 0.101$ & 1.00 & 0.86 & -0.54 \\
$T$ & $0.111 \pm 0.116$ & 0.86 & 1.00 & -0.83 \\
$U$ & $-0.016 \pm 0.088$ & -0.54 & -0.83 & 1.00 \\
\hline
\end{tabular}

Table 6. Results for the fit of the oblique parameters $S, T$ and $U$, excluding the information from $R_{b}$.

be used to constrain the parameter space of the model. The standard bounds on $S, T$ and $U[75]$ include in the global fit the ratio $R_{b}=\Gamma(Z \rightarrow b \bar{b}) / \Gamma(Z \rightarrow$ hadrons $)$, which incorporates quantum corrections to $\Gamma(Z \rightarrow b \bar{b})$ that are enhanced by the large value of the top quark mass $[80,81]$. Since the ratio $R_{b}$ receives also sizeable corrections from the additional scalars, we cannot use these bounds. Similarly to what has been done before for the CKM matrix, we first determine the oblique parameters, performing a combined fit with HEPfit of electroweak precision observables without $R_{b}$ [3], and then use the resulting values as inputs in our analysis of the MW model. The values of the oblique parameters that we have obtained from this fit are shown in table 6 .

The theoretical predictions for $S, T$ and $U$ in the MW model have been taken from ref. [11]. The oblique parameters depend on the masses of the new scalars and, therefore, provide important constraints on $m_{S}, \nu_{1}, \nu_{2}$ and $\nu_{3}$. Once, the oblique parameters have been fixed, we also include $R_{b}$ in the fit to the MW parameter space. The theoretical prediction of this observable, including the QCD corrections, has been taken from ref. [12]. The ratio $R_{b}$ will basically constrain $\eta_{U}$ because, like for the flavour observables, the new physics contribution comes from one-loop diagrams involving virtual charged scalars, coupled to heavy quarks.

\section{Results}

\subsection{Theoretical constraints}

As mentioned before, we have imposed that this model should satisfy perturbative unitarity and be well defined up to, at least, $3 \mathrm{TeV}$. These conditions generate bounds on all the parameters of the scalar potential that, in some cases, can be stronger than the ones 

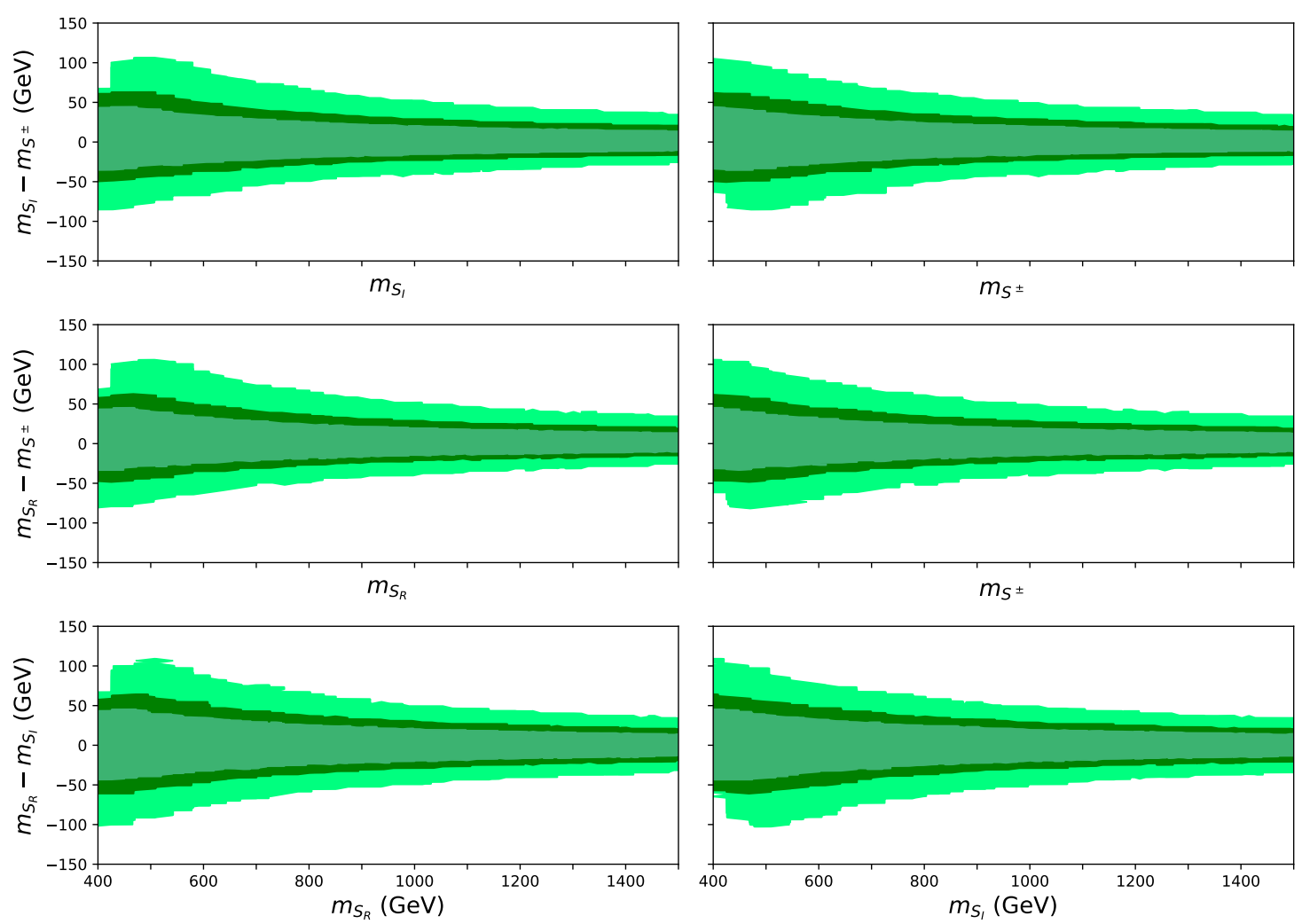

Figure 1. Theoretical constraints on the mass splittings, as functions of the scalar masses. The different coloured regions correspond to imposing RG stability up to an UV mass scale of 3 or $5 \mathrm{TeV}$, and imposing perturbative unitarity conditions above $2.5 \mathrm{TeV}$ or $1.5 \mathrm{TeV}$.

obtained from experimental data. For instance, the quartic couplings are not constrained experimentally but the theoretical requirements imply that none of them can be, in modulus, higher than 6 . The theoretical constraints can be translated to physical observables like the mass differences, which depend on these parameters. In figure 1 we show the theoretical constraints on the scalar mass differences for two choices of the UV scale, $3 \mathrm{TeV}$ and $5 \mathrm{TeV}$. Obviously, requiring the model to be valid up to a higher scale results in stronger bounds. The figure exhibits also the importance of perturbative unitarity, showing how the limits get weakened if the scale above which we impose it is chosen to be higher.

The large impact of these theoretical constraints can be better appreciated in figure 8 , which displays the bounds they impose on the $\mu_{n}$ and $\nu_{n}$ potential parameters, in the form of two-dimensional correlated plots, compared with the final results from the global fit, including all constraints, that we will later discuss. 

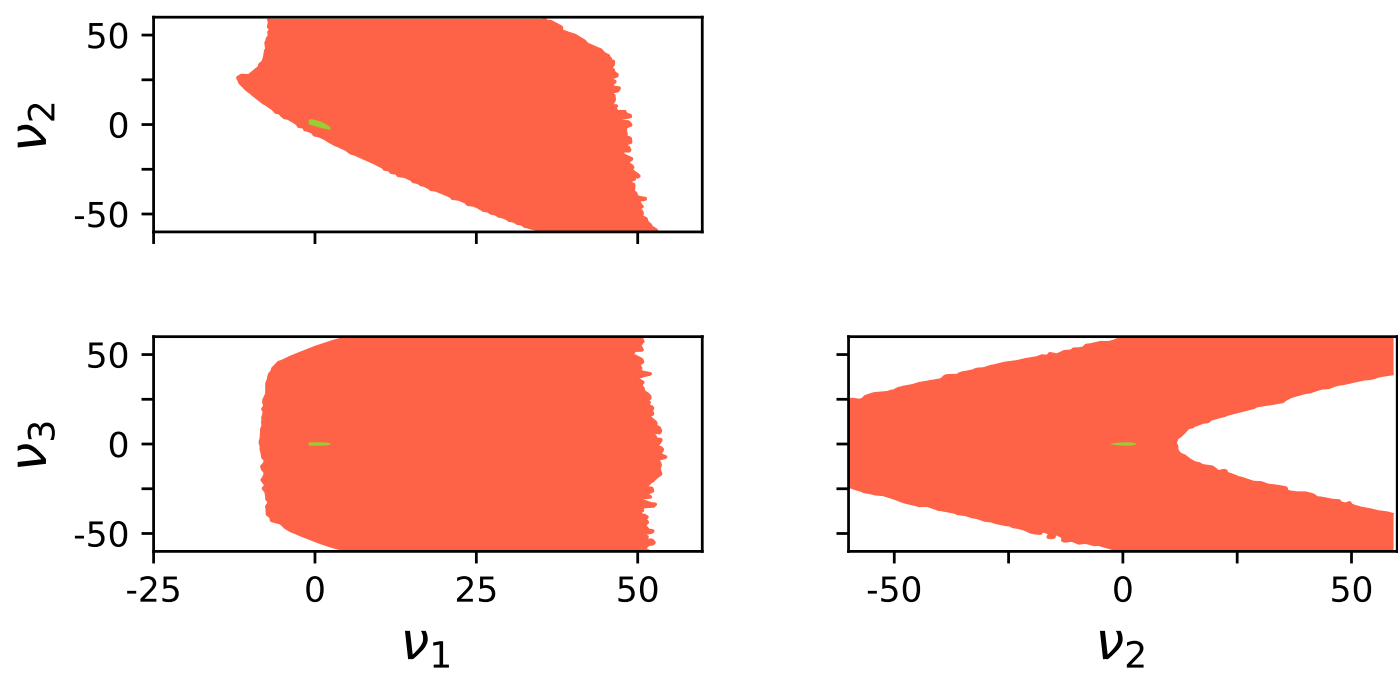

Figure 2. Two-dimensional constraints on $\nu_{1}, \nu_{2}$ and $\nu_{3}$ from Higgs signal strengths (red), compared with the theoretically allowed regions (green), assuming RG stability up to $3 \mathrm{TeV}$.

\subsection{Experimental constraints}

As we have shown before, using theoretical arguments we can constrain the parameter space of all the new quartic couplings that appear in the scalar potential. In this section we will use experimental measurements in order to constrain the masses of the coloured scalars and their Yukawa couplings. But first of all let us consider the observables that constrain also the parameters of the potential and let us compare the results with the ones obtained from theoretical requirements.

In figure 2 we show the constraints on $\nu_{1}, \nu_{2}$ and $\nu_{3}$ emerging from the Higgs signal strengths and compare them with the theoretically allowed regions, imposing RG stability up to $3 \mathrm{TeV}$. Clearly the theoretical restrictions are much stronger than the Higgs constraints so, a priori, one could think that the latter will be irrelevant in the global fit. However, the addition of these experimental observables will have some effect on the global fit, as we will show later. The fact that the constraints from the Higgs signal strengths, alone, are so weak is an expected behaviour, given that the coloured scalars only start to contribute to them at the one-loop level.

The oblique parameters constrain the scalar mass splittings, bounding the combinations of quartic couplings $\nu_{2} \pm 2 \nu_{3}$ that appear in eq. (2.2). This is clearly seen in the narrow allowed regions (orange) displayed in figure 3 . At large values of $\nu_{2,3}$, the oblique parameters impose that $\nu_{2} \approx \pm 2 \nu_{3}$ in order to reduce the splitting between the charged 


$\begin{array}{lll}95 \% \text { STU } & \text { Theo 3TeV Theo 5TeV }\end{array}$

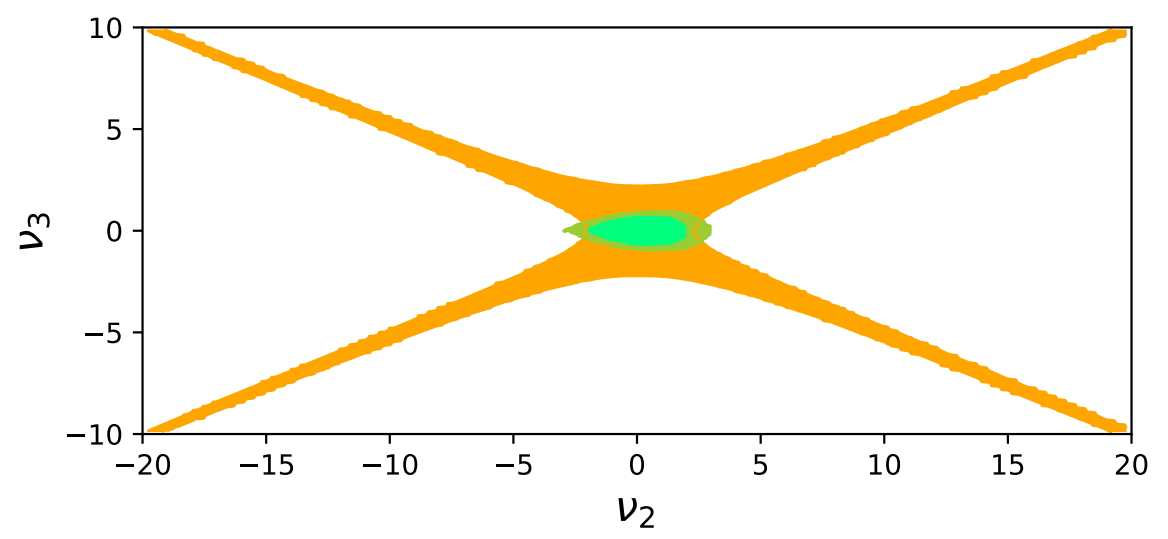

Figure 3. Allowed two-dimensional regions of $\nu_{2}$ and $\nu_{3}$ from the oblique parameters (orange, $95 \%$ probability) and from theoretical constraints (green) with the NLO+ approximation. The darker and lighter green areas correspond to requiring RG stability up to 3 and $5 \mathrm{TeV}$, respectively.

scalar and either the CP-odd or CP-even neutral scalars. The oblique bounds are relevant even when we consider the theoretical constraints up to $5 \mathrm{TeV}$, but they are specially important when we only require RG stability up to $3 \mathrm{TeV}$. Indeed, combining both constraints we can obtain a harder restriction on these parameters for the $3 \mathrm{TeV}$ case.

The absolute mass scale $m_{S}$ is constrained by the electroweak ratio $R_{b}$, which is also sensitive to the Yukawa alignment parameter $\eta_{U}$. The $m_{S}-\eta_{U}$ plane is also constrained by the two flavour observables that we have considered: $B_{s}^{0}-\bar{B}_{s}^{0}$ mixing and $\operatorname{Br}\left(B_{s} \rightarrow\right.$ $\mu^{+} \mu^{-}$). The left panel of figure 4 displays the regions allowed by each of these observables, individually, at $95 \%$ probability. The mass difference between $B_{s}^{0}$ and $\bar{B}_{s}^{0}$ turns out to provide the strongest bounds, although they are quite similar to the ones from $\operatorname{Br}\left(B_{s} \rightarrow\right.$ $\left.\mu^{+} \mu^{-}\right)$. Furthermore, since the three observables exhibit a similar dependence on these parameters, the combined constraints obtained with the three observables together are not much better than the ones given by just $\Delta M_{B_{s}}$ or $\operatorname{Br}\left(B_{s} \rightarrow \mu^{+} \mu^{-}\right)$.

The $m_{S}-\eta_{U}$ plane is also constrained by the direct searches, as shown in the right panel of figure 4. In this figure we have only included searches with at least one top quark in the final state because those are the channels generating the most interesting constraints. Since the figure does not include any observable constraining the scalar mass differences, we have set these mass splittings to zero. Doing so, we are able to totally exclude values of $m_{S}$ (equal in this case to the physical scalar masses) smaller than $1.1 \mathrm{TeV}$, for any value of the other parameters, with a probability of $95 \%$. The plot shows also the additional constraints obtained on this plane from flavour observables. 
In figure 5 we display the analogous constraints emerging from direct searches in channels without top quarks in the final state. Relevant bounds are only obtained at small values of $\eta_{U}$ or for high values of $\eta_{D}$. This is to be expected because those are the parameter regions where the scalar decays into top quarks are suppressed and, therefore, the branching ratios for the other channels are increased. The decay channels to top quarks are of course the dominant ones outside these particular regions of the parameter space, and decay modes without top quarks become irrelevant for large values of $\eta_{U}$ or small values of $\eta_{D}$.

Similar features are found in the $m_{S}-\eta_{D}$ plane, where the bound $m_{S}>1.1 \mathrm{TeV}$ is also obtained with a $95 \%$ probability from direct searches in channels with top-quark production. This could be, in principle, a bit surprising because one could naively expect that for very small values of $\eta_{U}$ those searches should not generate any constraint. This is true for the channels in which a neutral scalar decays to a $t \bar{t}$ pair, but not for a charged scalar decaying to $t \bar{b}$, a process which also depends on $\eta_{D}$. Indeed, in the right panel of figure 4 , one observes that the lower bound on $m_{S}$ decreases when $\eta_{U}$ approaches zero. This is because all channels with neutral scalars become irrelevant in that limit, but the information from charged-scalar channels is still good enough to generate a quite strong constraint. Therefore, as long as $\eta_{U}$ and $\eta_{D}$ are not both extremely close to zero, we obtain good constraints on $m_{S}$. Since the MW model is motivated by MFV, the particular region where the two quark Yukawa couplings are both zero does not seem to have much theoretical interest.

\subsection{All constraints}

Once we have analysed the constraints emerging from individual observables, we can combine all of them into a global fit. The first interesting result is that we are able to find lower limits for the physical masses of our scalars and constraints on their mass splittings, as shown in figure 6 . Note that the mass differences between the different scalars are now restricted to be smaller than $25 \mathrm{GeV}$ with a $99.7 \%$ probability, if we impose RG stability and perturbative unitarity up to $3 \mathrm{TeV}$, and to be smaller than $20 \mathrm{GeV}$, with the same probability, when we go up to $5 \mathrm{TeV}$. This justifies a posteriori our approximation of not including in the analysis of direct searches the decays of the coloured scalars into another scalar plus a weak boson.

Another interesting result is shown in figure 7, where we plot again the constraints on the $m_{S}-\eta_{U}$ and $m_{S}-\eta_{D}$ planes. We can clearly see how $\eta_{D}$ is not constrained. On the other hand, $\left|\eta_{U}\right|$ cannot take values higher than 1.8, for masses of the scalars smaller than $1.5 \mathrm{TeV}$, within a probability of $95 \%$. The constraint on the scalar mass scale is mainly coming from direct searches including decays to top quarks. This is why for values of $\eta_{U}$ close to zero the lower bounds on $m_{S}$ are weaker. However, as mentioned before, even for very small values of $\left|\eta_{U}\right|$ the constraints remain strong. We have also performed some global fits in which we set $\eta_{U}=0$ or $\eta_{D}=0$. For the first case we found that the limits on the scalar masses are roughly $100 \mathrm{GeV}$ smaller, while for the second we found the same result than for the global fit in which we vary both parameters. Therefore, even in the hypothetical case that some symmetry would force one of the Yukawa couplings to vanish, one would still find important constraints on the scalar masses. 


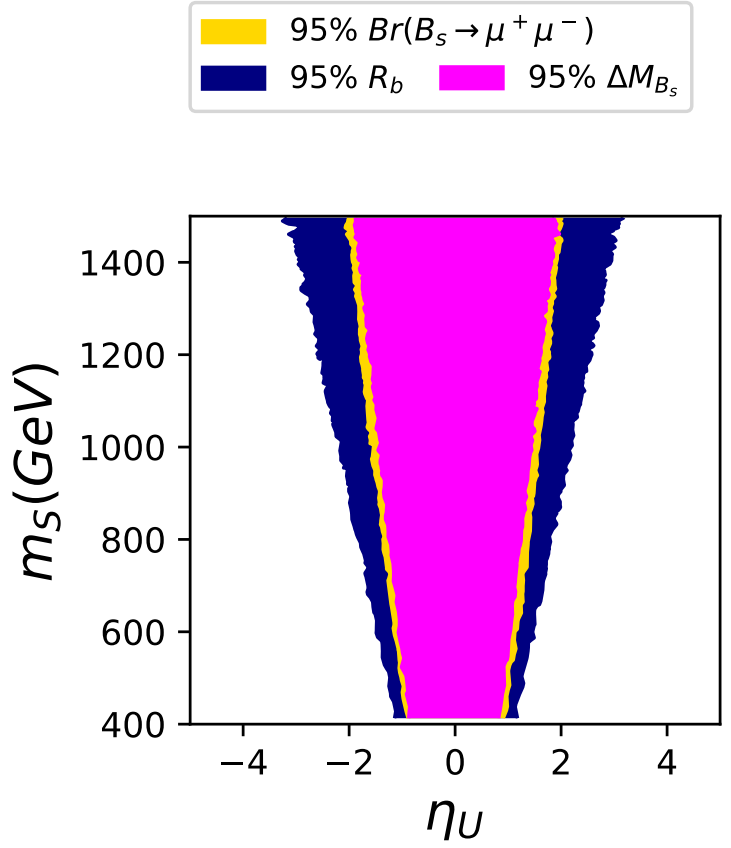

$95 \%$ DS t-quark

95\% Flavour

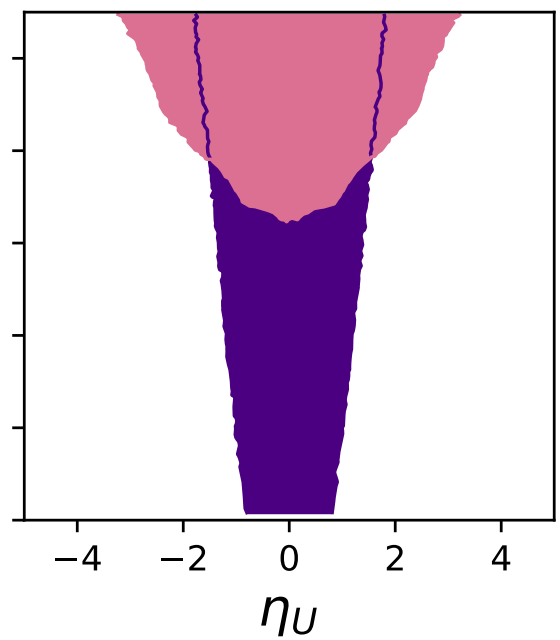

Figure 4. Experimental constraints on the $m_{S}-\eta_{U}$ plane. Left panel: allowed regions at $95 \%$ probability obtained from $R_{b}$ (blue), $\operatorname{Br}\left(B_{s} \rightarrow \mu^{+} \mu^{-}\right)$(yellow) and $\Delta M_{B_{s}}$ (magenta). Right panel: combined flavour constraints, compared with the limits from direct searches including top quarks, at $95 \%$ probability.

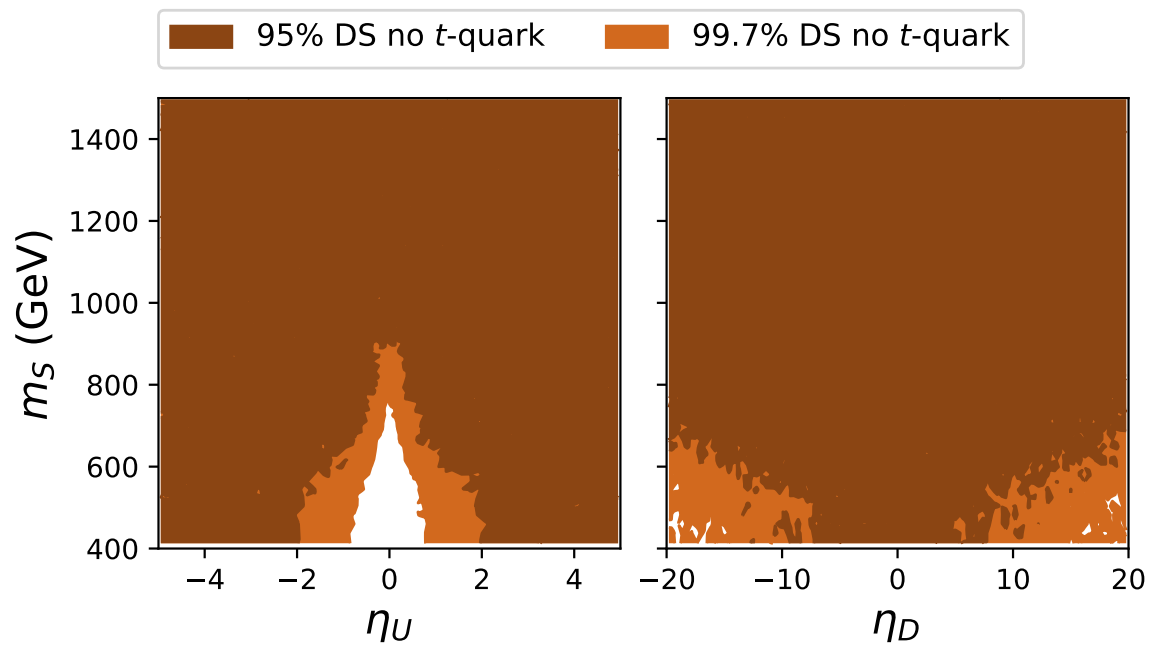

Figure 5. Constraints on the $m_{S}-\eta_{U}$ (left) and $m_{S}-\eta_{D}$ (right) planes, obtained from direct searches in channels without top quarks in the final state. 

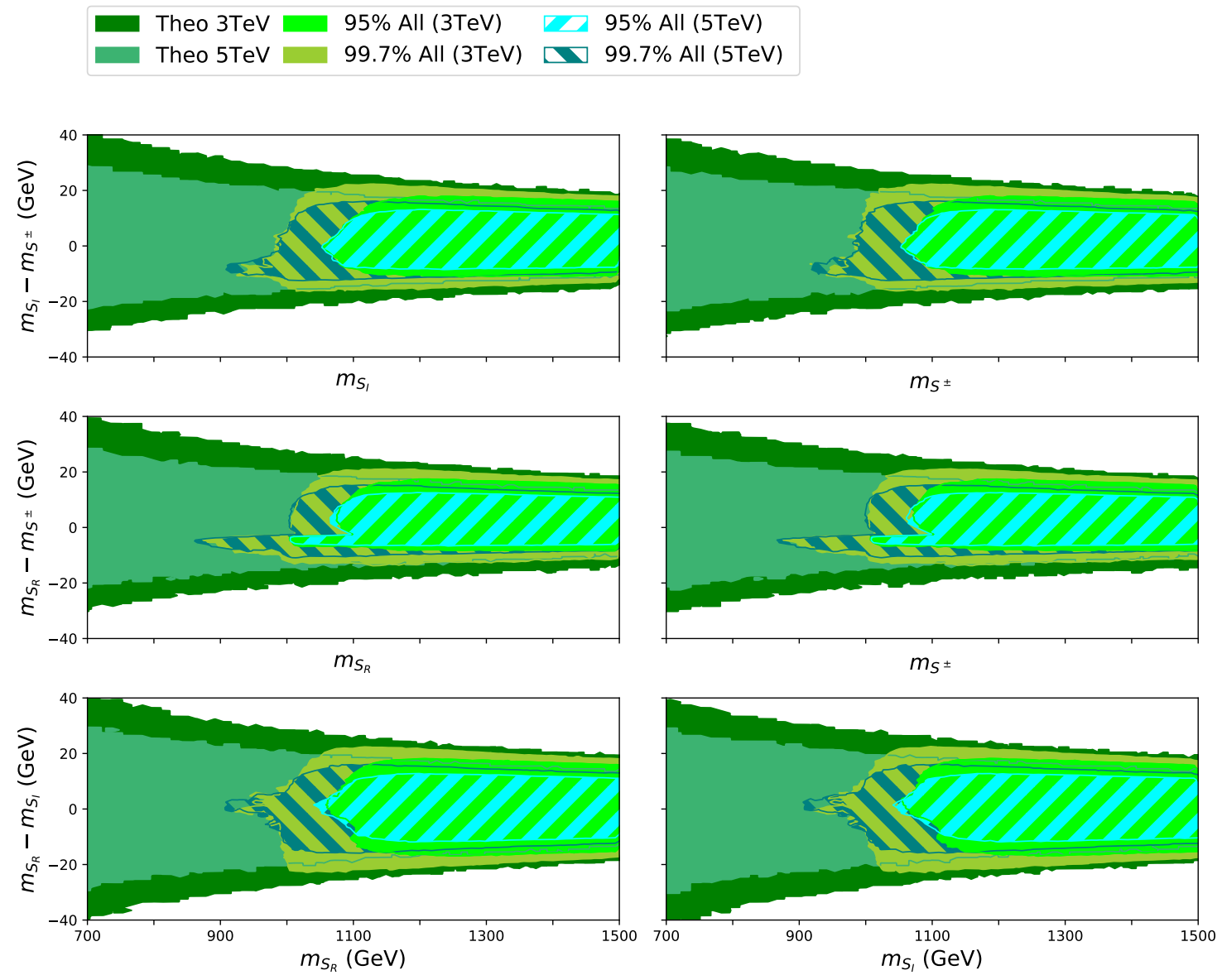

Figure 6. Comparison of theoretical and global constraints on the mass splittings, as a function of the different scalar masses, imposing RG stability and perturbative unitarity up to 3 and $5 \mathrm{TeV}$. For the global fit we plot the $95 \%$ and $99.7 \%$ regions.

The two-dimensional correlations among the quartic potential parameters are displayed in figure 8, which compares the limits emerging from the global fit with those obtained with only theoretical constraints, imposing RG stability and perturbative unitarity up to 3 and $5 \mathrm{TeV}$. The allowed regions from theoretical constraints must be understood as having $100 \%$ probability because they correspond to a discrete condition: a given point of the parameter space is either allowed or not. This introduces a small difference when comparing the theoretical and global limits, since the latter ones refer to a slightly smaller probability. Taking this into account, we can still see that $\nu_{1}$ and $\nu_{2}$ are slightly more constrained in the global fit, if RG stability is only imposed up to $3 \mathrm{TeV}$. Nevertheless, for the other observables the allowed regions remain almost invariant when including the experimental information. This is not a surprise because the measured observables depend on $\nu_{1}, \nu_{2}$ and $\nu_{3}$. The improvements introduced by the global fit are a consequence of adding the oblique parameters and, specially, the Higgs signal strengths. In order to check this, we performed a fit with only theory and the Higgs signal strengths and the results for $\nu_{1}$ and $\nu_{2}$ were 


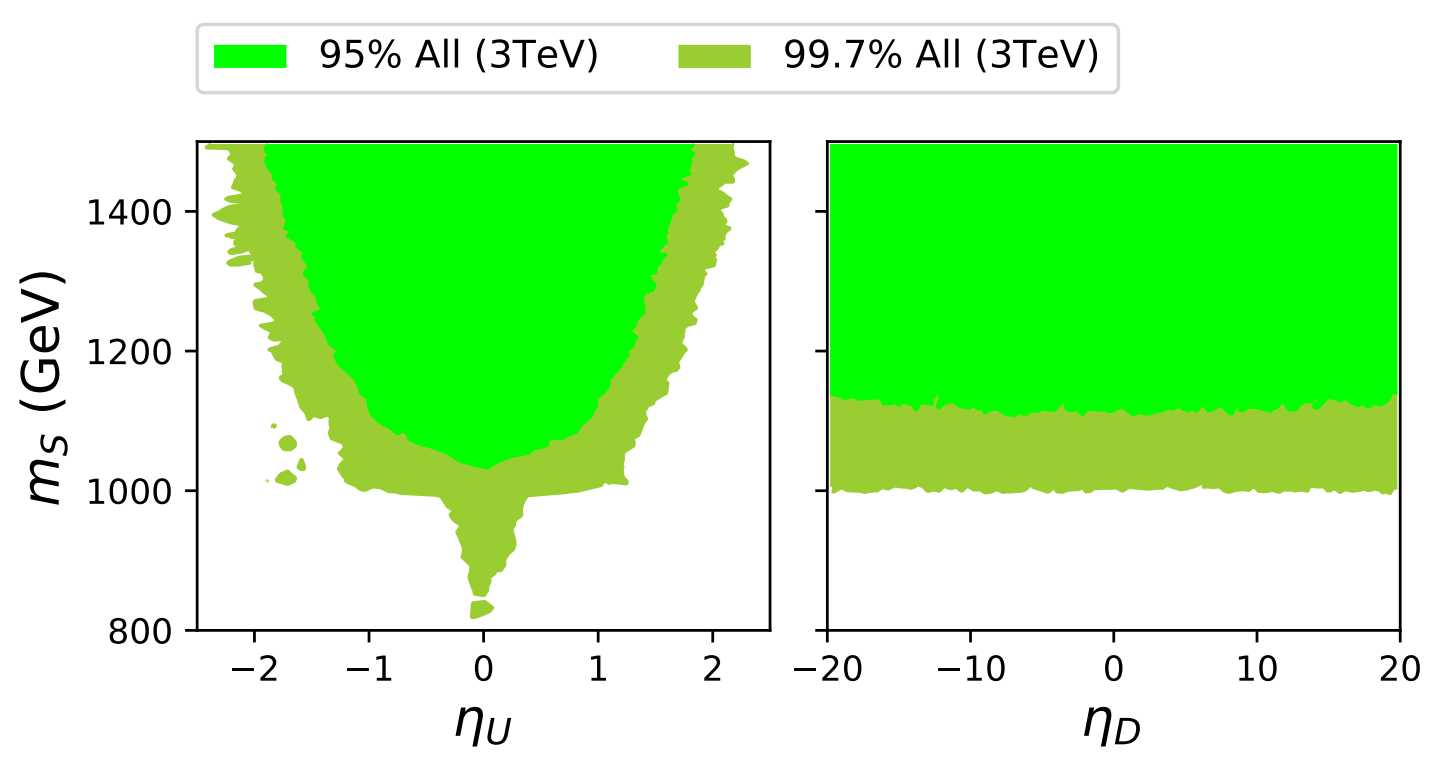

Figure 7. Allowed regions on the $m_{S^{-}} \eta_{U}$ (left) and $m_{S^{-}} \eta_{D}$ (right) planes from the global fit, at $95 \%$ and $99.7 \%$ probability, imposing RG stability up to $3 \mathrm{TeV}$ and perturbative unitarity at NLO+. Quite similar results are obtained, requiring RG stability up to only $1 \mathrm{TeV}$.

basically identical to the ones of the global fit. Indeed, although the Higgs signal strengths alone do not produce strong limits, combining them with the theoretical constraints, which reduce the allowed range of $\nu_{3}$, results in very good bounds on $\nu_{1}$ and $\nu_{2}$.

If RG stability is imposed up to $5 \mathrm{TeV}$, the current experimental measurements have a quite small impact in the allowed regions in figure 8 , which are mainly governed by the much stronger theoretical constraints.

Finally, in table 7 we present the marginalised allowed ranges for the parameters of the potential, from the global fit, with a probability of $95 \%$. As can be seen there, none of the quartic couplings can be, in modulus, higher than 5 within this probability.

\section{Summary}

In this work, we have presented the first global fit of the MW model. We have combined the more relevant theoretical and experimental constraints, including flavour and electroweak precision observables, Higgs signal strengths and direct searches. The theoretical constraints have a quite large impact on the scalar parameter space, specially when RG stability is imposed up to $5 \mathrm{TeV}$, providing bounds on all the scalar potential parameters. The current experimental information on the Higgs signal strengths and the oblique parameters gives an improved sensitivity to $\nu_{1}, \nu_{2}$ and $\nu_{3}$, which becomes relatively more important 


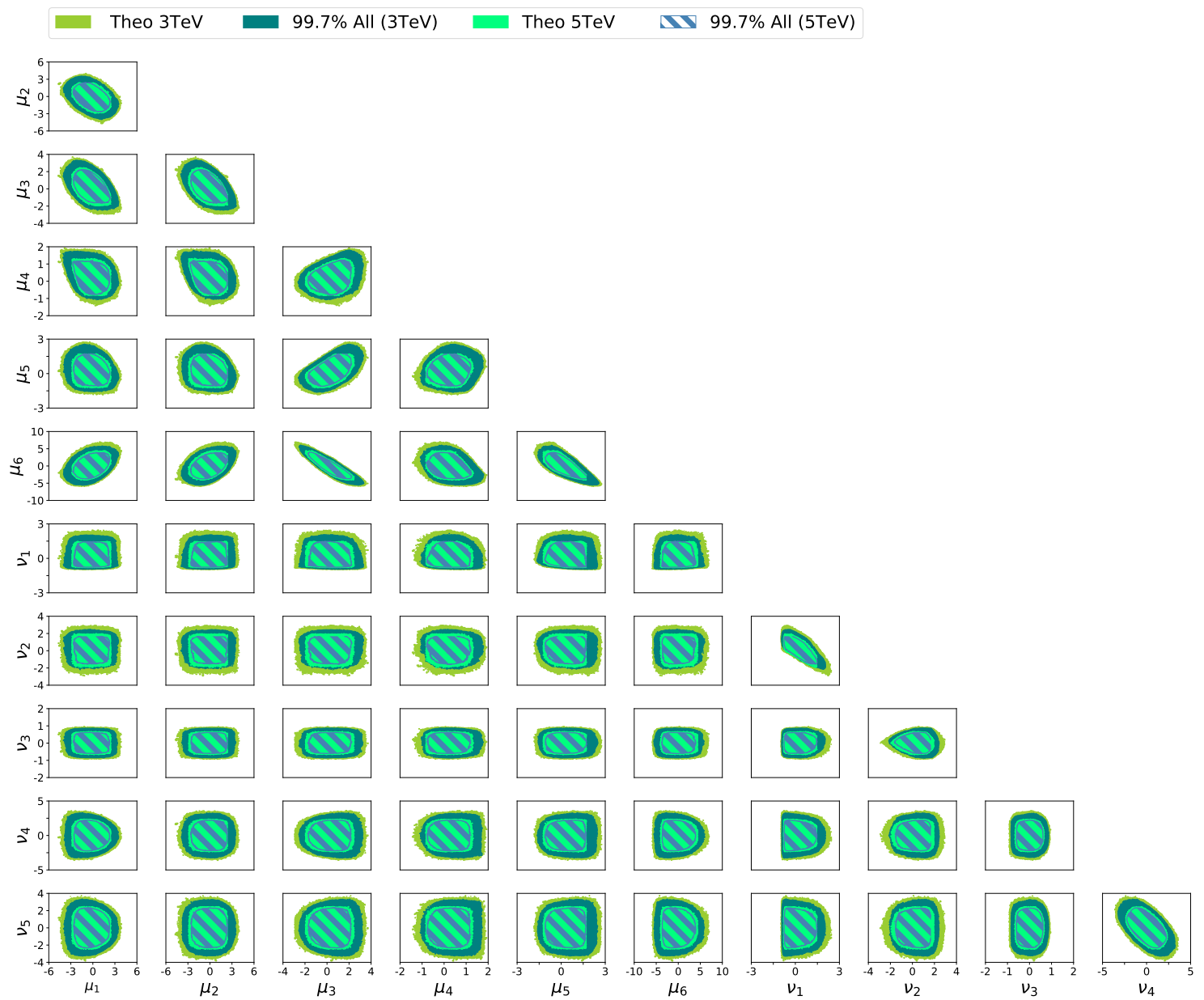

Figure 8. Two-dimensional correlations among the quartic couplings of the scalar potential. The allowed (99.7\% probability) regions from the global fit are compared with the results obtained with only theoretical constraints, imposing perturbative unitarity and RG stability up to 3 and $5 \mathrm{TeV}$.

\begin{tabular}{|c|c|c|}
\hline Variables & All $\mathbf{3 T e V}$ & All $\mathbf{5 T e V}$ \\
\hline$\mu_{1}$ & $(-2.9,2.3)$ & $(-1.3,1.5)$ \\
\hline$\mu_{2}$ & $(-2.7,2.6)$ & $(-1.8,1.8)$ \\
\hline$\mu_{3}$ & $(-1.6,2.4)$ & $(-1.0,1.6)$ \\
\hline$\mu_{4}$ & $(-0.60,1.35)$ & $(-0.44,0.90)$ \\
\hline$\mu_{5}$ & $(-1.0,1.9)$ & $(-0.7,1.3)$ \\
\hline$\mu_{6}$ & $(-4.6,3.5)$ & $(-2.9,2.4)$ \\
\hline$\nu_{1}$ & $(-0.8,1.4)$ & $(-0.66,0.97)$ \\
\hline$\nu_{2}$ & $(-1.2,2.0)$ & $(-0.95,1.47)$ \\
\hline$\nu_{3}$ & $(-0.65,0.70)$ & $(-0.45,0.49)$ \\
\hline$\nu_{4}$ & $(-2.4,2.4)$ & $(-1.7,1.6)$ \\
\hline$\nu_{5}$ & $(-1.6,1.5)$ & $(-1.7,1.6)$ \\
\hline
\end{tabular}

Table 7. Allowed ranges for the quartic potential parameters from the global fit, at $95 \%$ probability. 
if RG stability is only imposed up to $3 \mathrm{TeV}$. This allows for more stringent constraints on the scalar mass splittings.

The flavour observables and $R_{b}$ constrain the $m_{S^{-}} \eta_{U}$ plane, but all of them in the same direction. The strongest limits come from $\Delta M_{B_{s}}$ and $\operatorname{Br}\left(B_{s} \rightarrow \mu^{+} \mu^{-}\right)$, which combined, require that

$$
\left|\eta_{U}\right|<1.8
$$

for scalar masses smaller than $1.5 \mathrm{TeV}$, within a probability of $95 \%$.

A quite strong bound on the absolute mass scale $m_{S}$ emerges from the LHC data on direct searches for new scalars. The more sensitive channels, which involve the production of top quarks in the final state, imply that the masses of all coloured scalars must satisfy the bound

$$
m_{S^{ \pm}}, m_{S_{R}}, m_{S_{I}}>1.05 \mathrm{TeV}
$$

for any value of the other parameters, with a $95 \%$ probability. The global fit also restricts the scalar mass splittings to be smaller than $20 \mathrm{GeV}$, with a $95 \%$ probability, even when RG stability is only imposed up to $3 \mathrm{TeV}$.

As shown in ref. [28], even for tiny values of $\left|\eta_{U}\right|>10^{-7}$, one still finds a strong lower bound on the scalar masses, provided $\left|\eta_{D}\right|>10^{-5}$. These bounds can only be avoided for fermiophobic scalars with $\eta_{U} \approx \eta_{D} \approx 0$, such that their decay into a fermion-antifermion pair is highly suppressed. In that case, they would have a completely different experimental signature, since they would either decay into a lighter coloured scalar and a gauge boson or would behave as strongly-interacting long-lived particles. Although fermiophobic scalars are not compelling from the MFV point of view of the MW model, their phenomenology could be interesting by itself, but requires a more specific analysis that we postpone to future works.

The forthcoming Run3 of the LHC and its subsequent high-luminosity phase will provide much larger data samples, substantially increasing the sensitivity to coloured scalar particles. Mass scales up to $1.3 \mathrm{TeV}$ seem to be reachable with the expected luminosity of $3 \mathrm{ab}^{-1}$. The constraints on those potential parameters not related to scalar masses will remain, however, largely dependent on theoretical considerations, unless a real discovery of a colourful scalar state emerges from the data.

\section{Acknowledgments}

This work has been supported in part by the Spanish Government and ERDF funds from the EU Commission [grant FPA2017-84445-P], by the Generalitat Valenciana [grant Prometeo/2017/053] and by the COST Action CA16201 PARTICLEFACE. The work of V.M. is supported by the FPU doctoral contract FPU16/0191, funded by the Spanish Ministry of Universities.

Open Access. This article is distributed under the terms of the Creative Commons Attribution License (CC-BY 4.0), which permits any use, distribution and reproduction in any medium, provided the original author(s) and source are credited. 


\section{References}

[1] ATLAS collaboration, Observation of a new particle in the search for the Standard Model Higgs boson with the ATLAS detector at the LHC, Phys. Lett. B 716 (2012) 1 [arXiv:1207.7214] [INSPIRE].

[2] CMS collaboration, Observation of a new boson at a mass of $125 \mathrm{GeV}$ with the CMS experiment at the LHC, Phys. Lett. B 716 (2012) 30 [arXiv:1207.7235] [INSPIRE].

[3] O. Eberhardt, A.P. Martínez and A. Pich, Global fits in the aligned two-Higgs-doublet model, JHEP 05 (2021) 005 [arXiv: 2012.09200] [INSPIRE].

[4] H. Georgi and C. Jarlskog, A new lepton-quark mass relation in a unified theory, Phys. Lett. $B 86$ (1979) 297 [INSPIRE].

[5] I. Dorsner and P. Fileviez Perez, Unification versus proton decay in SU(5), Phys. Lett. B 642 (2006) 248 [hep-ph/0606062] [INSPIRE].

[6] P. Fileviez Perez and C. Murgui, Renormalizable SU(5) unification, Phys. Rev. D 94 (2016) 075014 [arXiv: 1604.03377] [INSPIRE].

[7] S. Bertolini, L. Di Luzio and M. Malinsky, Light color octet scalars in the minimal SO(10) grand unification, Phys. Rev. D 87 (2013) 085020 [arXiv:1302.3401] [INSPIRE].

[8] A.V. Manohar and M.B. Wise, Flavor changing neutral currents, an extended scalar sector, and the Higgs production rate at the CERN LHC, Phys. Rev. D 74 (2006) 035009 [hep-ph/0606172] [INSPIRE].

[9] M.I. Gresham and M.B. Wise, Color octet scalar production at the LHC, Phys. Rev. D 76 (2007) 075003 [arXiv:0706.0909] [InSPIRE].

[10] M. Gerbush, T.J. Khoo, D.J. Phalen, A. Pierce and D. Tucker-Smith, Color-octet scalars at the CERN LHC, Phys. Rev. D 77 (2008) 095003 [arXiv:0710.3133] [InSPIRE].

[11] C.P. Burgess, M. Trott and S. Zuberi, Light octet scalars, a heavy Higgs and minimal flavour violation, JHEP 09 (2009) 082 [arXiv:0907.2696] [INSPIRE].

[12] G. Degrassi and P. Slavich, QCD corrections in two-Higgs-doublet extensions of the Standard Model with minimal flavor violation, Phys. Rev. D 81 (2010) 075001 [arXiv:1002.1071] [INSPIRE].

[13] X.-G. He and G. Valencia, An extended scalar sector to address the tension between a fourth generation and Higgs searches at the LHC, Phys. Lett. B 707 (2012) 381 [arXiv:1108.0222] [INSPIRE].

[14] B.A. Dobrescu, G.D. Kribs and A. Martin, Higgs underproduction at the LHC, Phys. Rev. D 85 (2012) 074031 [arXiv:1112.2208] [INSPIRE].

[15] Y. Bai, J. Fan and J.L. Hewett, Hiding a heavy Higgs boson at the 7 TeV LHC, JHEP 08 (2012) 014 [arXiv:1112.1964] [INSPIRE].

[16] J.M. Arnold and B. Fornal, Color octet scalars and high $p_{T}$ four-jet events at LHC, Phys. Rev. D 85 (2012) 055020 [arXiv: 1112.0003] [INSPIRE].

[17] G.D. Kribs and A. Martin, Enhanced di-Higgs production through light colored scalars, Phys. Rev. D 86 (2012) 095023 [arXiv: 1207.4496] [INSPIRE].

[18] M. Reece, Vacuum instabilities with a wrong-sign Higgs-gluon-gluon amplitude, New J. Phys. 15 (2013) 043003 [arXiv: 1208.1765] [INSPIRE]. 
[19] J. Cao, P. Wan, J.M. Yang and J. Zhu, The SM extension with color-octet scalars: diphoton enhancement and global fit of LHC Higgs data, JHEP 08 (2013) 009 [arXiv:1303.2426] [INSPIRE].

[20] X.-G. He, H. Phoon, Y. Tang and G. Valencia, Unitarity and vacuum stability constraints on the couplings of color octet scalars, JHEP 05 (2013) 026 [arXiv: 1303.4848] [INSPIRE].

[21] X.-D. Cheng, X.-Q. Li, Y.-D. Yang and X. Zhang, $B_{s, d}-\bar{B}_{s, d}$ mixings and $B_{s, d} \rightarrow \ell^{+} \ell^{-}$decays within the Manohar-Wise model, J. Phys. G 42 (2015) 125005 [arXiv:1504.00839] [INSPIRE].

[22] L. Cheng and G. Valencia, Two Higgs doublet models augmented by a scalar colour octet, JHEP 09 (2016) 079 [arXiv: 1606.01298] [INSPIRE].

[23] R. Martinez and G. Valencia, Top and bottom tensor couplings from a color octet scalar, Phys. Rev. D 95 (2017) 035041 [arXiv:1612.00561] [INSPIRE].

[24] A. Hayreter and G. Valencia, LHC constraints on color octet scalars, Phys. Rev. D 96 (2017) 035004 [arXiv: 1703.04164] [INSPIRE].

[25] L. Cheng, O. Eberhardt and C.W. Murphy, Novel theoretical constraints for color-octet scalar models, Chin. Phys. C 43 (2019) 093101 [arXiv: 1808. 05824] [INSPIRE].

[26] A. Hayreter and G. Valencia, Color-octet scalar decays to a gluon and an electroweak gauge boson in the Manohar-Wise model, Phys. Rev. D 102 (2020) 115033 [arXiv:1810.04048] [INSPIRE].

[27] H. Gisbert and J. Ruiz Vidal, Improved bounds on heavy quark electric dipole moments, Phys. Rev. D 101 (2020) 115010 [arXiv:1905.02513] [InSPIRE].

[28] V. Miralles and A. Pich, LHC bounds on colored scalars, Phys. Rev. D 100 (2019) 115042 [arXiv: 1910.07947] [INSPIRE].

[29] V. Miralles, Global fits in the coloured scalar model, Nucl. Part. Phys. Proc. 309-311 (2020) 63 [INSPIRE].

[30] J. De Blas et al., HEPfit: a code for the combination of indirect and direct constraints on high energy physics models, Eur. Phys. J. C 80 (2020) 456 [arXiv:1910.14012] [InSPIRE].

[31] R.S. Chivukula and H. Georgi, Composite technicolor Standard Model, Phys. Lett. B 188 (1987) 99 [INSPIRE].

[32] G. D'Ambrosio, G.F. Giudice, G. Isidori and A. Strumia, Minimal flavor violation: an effective field theory approach, Nucl. Phys. B 645 (2002) 155 [hep-ph/0207036] [INSPIRE].

[33] A. Caldwell, D. Kollar and K. Kroninger, BAT: the Bayesian Analysis Toolkit, Comput. Phys. Commun. 180 (2009) 2197 [arXiv: 0808. 2552] [INSPIRE].

[34] V. Cacchio, D. Chowdhury, O. Eberhardt and C.W. Murphy, Next-to-leading order unitarity fits in two-Higgs-doublet models with soft $Z_{2}$ breaking, JHEP 11 (2016) 026 [arXiv: 1609.01290] [INSPIRE].

[35] S. Gori, C. Grojean, A. Juste and A. Paul, Heavy Higgs searches: flavour matters, JHEP 01 (2018) 108 [arXiv: 1710.03752] [INSPIRE].

[36] D. Chowdhury and O. Eberhardt, Update of global two-Higgs-doublet model fits, JHEP 05 (2018) 161 [arXiv: 1711.02095] [INSPIRE].

[37] C.-W. Chiang, G. Cottin and O. Eberhardt, Global fits in the Georgi-Machacek model, Phys. Rev. D 99 (2019) 015001 [arXiv: 1807.10660] [INSPIRE]. 
[38] M. Farina, C. Mondino, D. Pappadopulo and J.T. Ruderman, New physics from high energy tops, JHEP 01 (2019) 231 [arXiv: 1811.04084] [INSPIRE].

[39] J. de Blas, O. Eberhardt and C. Krause, Current and future constraints on Higgs couplings in the nonlinear effective theory, JHEP 07 (2018) 048 [arXiv: 1803.00939] [INSPIRE].

[40] G. Durieux et al., The electro-weak couplings of the top and bottom quarks - global fit and future prospects, JHEP 12 (2019) 098 [Erratum ibid. 01 (2021) 195] [arXiv:1907.10619] [INSPIRE].

[41] V. Miralles, M.M. López, M.M. Llácer, A. Peñuelas, M. Perelló and M. Vos, The top quark electro-weak couplings after LHC run 2, arXiv:2107.13917 [INSPIRE].

[42] C.W. Murphy, NLO perturbativity bounds on quartic couplings in renormalizable theories with $\phi^{4}$-like scalar sectors, Phys. Rev. D 96 (2017) 036006 [arXiv:1702.08511] [InSPIRE].

[43] ATLAS collaboration, Measurement of Higgs boson production in the diphoton decay channel in pp collisions at center-of-mass energies of 7 and $8 \mathrm{TeV}$ with the ATLAS detector, Phys. Rev. D 90 (2014) 112015 [arXiv:1408.7084] [INSPIRE].

[44] CMS collaboration, Observation of the diphoton decay of the Higgs boson and measurement of its properties, Eur. Phys. J. C 74 (2014) 3076 [arXiv: 1407.0558] [InSPIRE].

[45] ATLAS collaboration, Measurement of the properties of Higgs boson production at $\sqrt{s}=13 \mathrm{TeV}$ in the $H \rightarrow \gamma \gamma$ channel using $139 \mathrm{fb}^{-1}$ of $p p$ collision data with the ATLAS experiment, Tech. Rep. ATLAS-CONF-2020-026, CERN, Geneva, Switzerland (2020).

[46] CMS collaboration, Measurements of Higgs boson production cross sections and couplings in the diphoton decay channel at $\sqrt{s}=13 \mathrm{TeV}$, JHEP 07 (2021) 027 [arXiv:2103.06956] [INSPIRE].

[47] ATLAS and CMS collaborations, Measurements of the Higgs boson production and decay rates and constraints on its couplings from a combined ATLAS and CMS analysis of the LHC pp collision data at $\sqrt{s}=7$ and $8 \mathrm{TeV}$, JHEP 08 (2016) 045 [arXiv:1606.02266] [INSPIRE].

[48] ATLAS collaboration, A search for the dimuon decay of the Standard Model Higgs boson with the ATLAS detector, Phys. Lett. B 812 (2021) 135980 [arXiv:2007.07830] [INSPIRE].

[49] CMS collaboration, Evidence for Higgs boson decay to a pair of muons, JHEP 01 (2021) 148 [arXiv: 2009. 04363] [INSPIRE].

[50] ATLAS collaboration, Evidence for the Higgs-boson Yukawa coupling to tau leptons with the ATLAS detector, JHEP 04 (2015) 117 [arXiv:1501.04943] [INSPIRE].

[51] CMS collaboration, Evidence for the $125 \mathrm{GeV}$ Higgs boson decaying to a pair of $\tau$ leptons, JHEP 05 (2014) 104 [arXiv: 1401.5041] [INSPIRE].

[52] ATLAS collaboration, A combination of measurements of Higgs boson production and decay using up to $139 \mathrm{fb}^{-1}$ of proton-proton collision data at $\sqrt{\mathrm{s}}=13 \mathrm{TeV}$ collected with the ATLAS experiment, Tech. Rep. ATLAS-CONF-2020-027, CERN, Geneva, Switzerland (2020).

[53] CMS collaboration, Combined Higgs boson production and decay measurements with up to $137 \mathrm{fb}^{-1}$ of proton-proton collision data at $\sqrt{s}=13 \mathrm{TeV}$, Tech. Rep. CMS-PAS-HIG-19-005, CERN, Geneva, Switzerland (2020).

[54] ATLAS collaboration, Observation and measurement of Higgs boson decays to $W W^{*}$ with the ATLAS detector, Phys. Rev. D 92 (2015) 012006 [arXiv:1412.2641] [INSPIRE]. 
[55] ATLAS collaboration, Study of $(W / Z) H$ production and Higgs boson couplings using $H \rightarrow W W^{*}$ decays with the ATLAS detector, JHEP 08 (2015) 137 [arXiv:1506.06641] [INSPIRE].

[56] CMS collaboration, Measurement of Higgs boson production and properties in the $W W$ decay channel with leptonic final states, JHEP 01 (2014) 096 [arXiv:1312.1129] [INSPIRE].

[57] ATLAS collaboration, Measurements of the Higgs boson production and decay rates and coupling strengths using pp collision data at $\sqrt{s}=7$ and $8 \mathrm{TeV}$ in the ATLAS experiment, Eur. Phys. J. C 76 (2016) 6 [arXiv:1507.04548] [inSPIRE].

[58] CMS collaboration, Search for a Higgs boson decaying into a $Z$ and a photon in pp collisions at $\sqrt{s}=7$ and $8 \mathrm{TeV}$, Phys. Lett. B 726 (2013) 587 [arXiv:1307.5515] [INSPIRE].

[59] ATLAS collaboration, A search for the $Z \gamma$ decay mode of the Higgs boson in pp collisions at $\sqrt{s}=13 \mathrm{TeV}$ with the ATLAS detector, Phys. Lett. B 809 (2020) 135754 [arXiv: 2005.05382] [INSPIRE].

[60] CMS collaboration, Search for the decay of a Higgs boson in the $\ell \ell \gamma$ channel in proton-proton collisions at $\sqrt{s}=13 \mathrm{TeV}$, JHEP 11 (2018) 152 [arXiv:1806.05996] [INSPIRE].

[61] ATLAS collaboration, Measurements of Higgs boson production and couplings in the four-lepton channel in pp collisions at center-of-mass energies of 7 and $8 \mathrm{TeV}$ with the ATLAS detector, Phys. Rev. D 91 (2015) 012006 [arXiv:1408.5191] [InSPIRE].

[62] CMS collaboration, Precise determination of the mass of the Higgs boson and tests of compatibility of its couplings with the Standard Model predictions using proton collisions at 7 and $8 \mathrm{TeV}$, Eur. Phys. J. C $\mathbf{7 5}$ (2015) 212 [arXiv:1412.8662] [INSPIRE].

[63] ATLAS collaboration, Higgs boson production cross-section measurements and their EFT interpretation in the $4 \ell$ decay channel at $\sqrt{s}=13 \mathrm{TeV}$ with the ATLAS detector, Eur. Phys. J. C 80 (2020) 957 [Erratum ibid. 81 (2021) 29] [Erratum ibid. 81 (2021) 398] [arXiv: 2004.03447] [INSPIRE].

[64] ATLAS collaboration, Search for heavy particles decaying into top-quark pairs using lepton-plus-jets events in proton-proton collisions at $\sqrt{s}=13 \mathrm{TeV}$ with the ATLAS detector, Eur. Phys. J. C 78 (2018) 565 [arXiv:1804.10823] [INSPIRE].

[65] ATLAS collaboration, Search for new phenomena in $t \bar{t}$ final states with additional heavy-flavour jets in pp collisions at $\sqrt{s}=13 \mathrm{TeV}$ with the ATLAS detector, Tech. Rep. ATLAS-CONF-2016-104, CERN, Geneva, Switzerland (2016).

[66] ATLAS collaboration, Search for new phenomena in events with same-charge leptons and b-jets in pp collisions at $\sqrt{s}=13 \mathrm{TeV}$ with the ATLAS detector, JHEP 12 (2018) 039 [arXiv: 1807.11883] [INSPIRE].

[67] ATLAS collaboration, Search for charged Higgs bosons decaying into a top quark and a bottom quark at $\sqrt{s}=13 \mathrm{TeV}$ with the ATLAS detector, JHEP 06 (2021) 145 [arXiv: 2102.10076] [INSPIRE].

[68] CMS collaboration, Search for neutral MSSM Higgs bosons decaying into a pair of bottom quarks, JHEP 11 (2015) 071 [arXiv:1506.08329] [INSPIRE].

[69] CMS collaboration, Search for narrow resonances in the b-tagged dijet mass spectrum in proton-proton collisions at $\sqrt{s}=8 \mathrm{TeV}$, Phys. Rev. Lett. 120 (2018) 201801 [arXiv: 1802.06149] [INSPIRE]. 
[70] CMS collaboration, Search for a narrow heavy decaying to bottom quark pairs in the $13 \mathrm{TeV}$ data sample, Tech. Rep. CMS-PAS-HIG-16-025, CERN, Geneva, Switzerland (2016).

[71] CMS collaboration, Search for beyond the standard model Higgs bosons decaying into a $b \bar{b}$ pair in pp collisions at $\sqrt{s}=13 \mathrm{TeV}$, JHEP 08 (2018) 113 [arXiv:1805.12191] [INSPIRE].

[72] CMS collaboration, Search for narrow and broad dijet resonances in proton-proton collisions at $\sqrt{s}=13 \mathrm{TeV}$ and constraints on dark matter mediators and other new particles, JHEP 08 (2018) 130 [arXiv: 1806.00843] [INSPIRE].

[73] ATLAS collaboration, A search for pair-produced resonances in four-jet final states at $\sqrt{s}=13 \mathrm{TeV}$ with the ATLAS detector, Eur. Phys. J. C 78 (2018) 250 [arXiv:1710.07171] [INSPIRE].

[74] J. Alwall et al., The automated computation of tree-level and next-to-leading order differential cross sections, and their matching to parton shower simulations, JHEP 07 (2014) 079 [arXiv: 1405.0301] [INSPIRE].

[75] Particle Data Group collaboration, Review of particle physics, PTEP 2020 (2020) 083C01 [INSPIRE].

[76] HPQCD collaboration, Improved $V_{c s}$ determination using precise lattice QCD form factors for $D \rightarrow K \ell \nu$, Phys. Rev. D 104 (2021) 034505 [arXiv: 2104.09883] [INSPIRE].

[77] Particle Data Group collaboration, Review of particle physics, Phys. Rev. D 98 (2018) 030001 [INSPIRE].

[78] M.E. Peskin and T. Takeuchi, A new constraint on a strongly interacting Higgs sector, Phys. Rev. Lett. 65 (1990) 964 [INSPIRE].

[79] M.E. Peskin and T. Takeuchi, Estimation of oblique electroweak corrections, Phys. Rev. D 46 (1992) 381 [INSPIRE].

[80] J. Bernabeu, A. Pich and A. Santamaria, $\Gamma(Z \rightarrow b \bar{b})$ : a signature of hard mass terms for a heavy top, Phys. Lett. B 200 (1988) 569 [InSPIRE].

[81] J. Bernabeu, A. Pich and A. Santamaria, Top quark mass from radiative corrections to the $Z \rightarrow b \bar{b}$ decay, Nucl. Phys. B 363 (1991) 326 [InSPIRE]. 